\title{
Optimal Low-Power Design of a Multicell Multiuser Massive MIMO System at 3.7 GHz for 5G Wireless Networks
}

\author{
Michel Matalatala (D), Margot Deruyck, Emmeric Tanghe (D), \\ Luc Martens, and Wout Joseph \\ Ghent University/IMEC, Department of Information Technology, Technologiepark-Zwijnaarde 15, B-9052 Ghent, Belgium \\ Correspondence should be addressed to Michel Matalatala; michel.matalatala@ugent.be
}

Received 18 June 2018; Revised 21 September 2018; Accepted 27 September 2018; Published 23 October 2018

Academic Editor: Xianfu Lei

Copyright (c) 2018 Michel Matalatala et al. This is an open access article distributed under the Creative Commons Attribution License, which permits unrestricted use, distribution, and reproduction in any medium, provided the original work is properly cited.

\begin{abstract}
Massive MIMO techniques are expected to deliver significant performance gains for the future wireless communication networks by improving the spectral and the energy efficiencies. In this paper, we propose a method to optimize the positions, the coverage, and the energy consumption of the massive MIMO base stations within a suburban area in Ghent, Belgium, while meeting the low power requirements. The results reveal that massive MIMO provides better performances for the crowded scenario where users' mobility is limited. With 256 antennas, a massive MIMO base station can simultaneously multiplex 18 users at the same timefrequency resource while consuming 8 times less power and providing 200 times more capacity than a $4 \mathrm{G}$ reference network for the same coverage. Moreover, a pilot reuse pattern of 3 is recommended in a multiuser multicell environment to obtain a good tradeoff between the high spectral efficiency and the low power requirement.
\end{abstract}

\section{Introduction}

The demand for reliable high throughput wireless communication links requires an unprecedented key technology to be enabled in the future fifth-generation wireless systems (5G). Both academia and industry have identified massive Multiple Input Multiple Output (MIMO) as an indispensable technology that can support high spectral efficiencies to cope with the requested high throughput, providing impressive energy efficiency at the same time $[1,2]$. The key distinguishing feature of massive MIMO from the conventional MIMO resides in the use of a very large number of antennas at the base stations (BS) and the multiplexing and demultiplexing of users data based on the measured propagation characteristics rather than on assumed propagation characteristics. The use of many antennas at the base BS brings about favorable propagation conditions and renders the simplest types of signal processing (both linear decoding in the uplink and linear precoding in the downlink) exceedingly effective. Moreover, it permits the same quality of service with less radiated power [3-5] compared to $4 \mathrm{G}$.
However, there are technical challenges in practical design which require to be tackled with appropriate architectures schemes: signal modeling, performance analysis, electromagnetic field (EMF), spectral efficiency (SE), and energy efficiency. Various recent works have addressed these topics. In $[6,7]$, the authors derived the achievable SE of a massive discrete Fourier transform (DFT) based low-cost hybrid architecture assuming they have perfect knowledge of channel state information (CSI) and zero-forcing (ZF) processing at the BS. They showed that it is lower than the achievable SE obtained with the hybrid architecture with ideal and quantized phase shifters. The work in [8] investigated a single-cell multiuser system consisting of a BS with a uniform rectangular array of antennas and showed that the achievable SE logarithmically increases with the number of antennas. Moreover, they improved the obtained achievable SE thanks to a user scheduling scheme which relies on the feedback of users' angle of departure (AoD) information. Other recent studies have assessed the EMF exposure for the base stations using massive MIMO [9-11]. While [9, 11] proposed statistical-based models for a realistic assessment 
of the EMF exposure for $5 \mathrm{G}$ base stations employing massive MIMO to meet the EMF regulations requirements, the authors in [10] discussed the necessity and the impacts of considering the EMF exposure constraints on the planning of $5 \mathrm{G}$ cellular network.

In this study, we focus on the design of a multiuser multicell massive MIMO system which provides higher throughput to the users, while meeting the low power consumption requirements. Few recent related works in [12, 13] addressed the design of macrocellular massive MIMO networks where the energy efficiency (EE) is maximized for a given coverage of the users (95\%). In [12], based on an assumed number of network cells, the authors investigated how the optimal number of users $K$ depends on the number of BS antennas $M$ and other parameters such as the precoding scheme, to maximize the EE. The paper [13] maximized the EE of a multicell massive MIMO system while optimizing the number of users served simultaneously and the total radiated power. With 15 users served simultaneously, the authors could achieve a total EE which is 1000 times greater than that of a Long Term Evolution (LTE). The main contribution of this paper consists in designing a multicell massive MIMO network in a realistic environment while optimizing the placements of the $5 \mathrm{G}$ BSs with the objective of minimizing the overall power consumption. To this end, we propose as novelty a simulation-based tool which optimizes the set of realistic possible locations of BSs retrieved from a mobile operator in Ghent, Belgium, so that the designed cellular massive MIMO network responds to the requested bit rate of the users, while meeting the coverage probability (at least 95\% users) and power consumption requirements. The relevant performance parameters of the designed networks are assessed and compared to the LTE network. To the best of our knowledge, none of the related existing works investigated the optimization of BS positions, in the context of multicell massive MIMO.

The remainder of this paper is organized as follows. Section 2 discusses the multicell massive MIMO system in the context of $5 \mathrm{G}$ and the related assumptions. In Section 3, the massive MIMO network design process is thoroughly described and finally Section 4 presents the results obtained with the capacity-based deployment tool with respect to the assumed massive MIMO scenarios. We then provide the final conclusions in Section 5.

Notation. Throughout the paper, we consider now the convention whereby matrices and vectors are expressed as upper and lower case boldface letters, respectively.

\section{Multicell Massive MIMO System Model Description}

The massive MIMO system model consists of multiple base stations, each equipped with a large number of antennas, $M$, operating phase-coherently. Each base station serves many single-antenna users $K$ in its associated cell at the same time. However, the different base stations do not cooperate. Each user is associated and covered by one of the BSs.
The number of BS antennas $M$ and maximum served users $K_{\max }$ are assumed, while the number of active users $\mathrm{K}$ served by a given BS results from the BS-user association algorithms. To achieve this, we assume that the network operates in time division duplex (TDD) mode to benefit from its channel reciprocity properties. The uplink (UL) pilots are used to estimate both the uplink and downlink (DL) channels on the BS side. We consider that these channels are constant during the coherence time interval of length $\tau_{c}$ expressed in symbols and change independently every interval. In each time interval, $\tau_{p}$ symbols are used for channel estimation in uplink and $\tau_{c}-\tau_{p}$ symbols are dedicated for the data transmission. In this paper, we focus on the DL data transmission. The expressions of the Signal to Interference plus Noise Ratio (SINR) and achievable sumrate (or capacity) considered throughout this work assume the simple linear precoding technique denoted maximum ratio combining/maximum ratio transmission (MRC/MRT). However, advanced processing techniques like zero-forcing (ZF) can also be used.

Throughout this paper, we assume that all operations take place in the coherence interval: uplink pilot transmission, uplink payload transmission, and downlink transmission [16]. Downlink pilots transmission is not considered. Therefore, both the uplink training and downlink transmission time are less than or equal to the channel coherence interval.

Moreover, the small-scale fading coefficients $\boldsymbol{h}_{\boldsymbol{i} j \boldsymbol{l} \boldsymbol{m}}$, from user $i$ in cell $j$ to the $m^{\text {th }}$ antenna at BS $l$, are assumed independent and identically distributed (i.i.d) Rayleigh fading [17] and have to be estimated from the received pilot signals. The massive MIMO measurements based on experimental data conducted in [18] agree with this assumption. Therefore, $\boldsymbol{h}_{\boldsymbol{i} \mathbf{j} \boldsymbol{m}}$ follows a complex Gaussian distribution with zero mean and unit variance. The large-scale fading which changes slowly over a period of time captures the effects of the path loss, the geometric attenuation, and the shadowing and is independent of the frequency over the $M$ BS antennas. It is denoted by $\beta_{i j l}$ [19], with the subscripts $i, j$, and $l$ referring to the user, the serving cell, and the base station, respectively. We assume that the large-scale fading is perfectly known.

Based on the above assumptions, the system can be modeled by the propagation factor between the $m^{\text {th }}$ antenna of the base station at the $j^{\text {th }}$ cell and the $k^{\text {th }}$ user at the $l^{\text {th }}$ cell as follows [19]:

$$
\boldsymbol{g}_{j k l}=\sqrt{\beta_{j k l}} \cdot \boldsymbol{h}_{j k l}
$$

where $\boldsymbol{g}_{\boldsymbol{j} \boldsymbol{k} \boldsymbol{l}}$ is the propagation factor between the $m^{\text {th }}$ antenna of the base station at $j^{\text {th }}$ cell and the $k^{\text {th }}$ user at the $l^{\text {th }}$ cell; $\boldsymbol{h}_{\boldsymbol{j} \boldsymbol{k} \boldsymbol{l}}$ is an independently identically distributed (i.i.d) fast Rayleigh fading coefficient from the $k^{\text {th }}$ user to the $m^{\text {th }}$ antenna of the base station with zero mean and unit variance; $\sqrt{\beta_{j k l}}$ represents the large-scale fading that takes into account the effects of the geometric attenuation, the shadow fading, and the path loss.

In normal massive MIMO TDD operation, the BS receives the pilot signals from the users during the uplink 
phase and proceeds with an estimation of the propagation channel matrix in (1) at each antenna by means of a linear estimation theory such as the minimum mean square error estimator (MMSE) [5]. During the downlink phase, the BS precodes the signals to all the $K$ users in the same time-frequency resource based on the propagation matrix estimated in the uplink. To benefit from the advantages of massive MIMO, it is necessary to obtain an accurate channel state information (CSI) at the BS side. This is hardly achievable in practice in a multicell environment since the transmission from the $j^{\text {th }}$ BS in the $l^{\text {th }}$ cell to its associated $k^{\text {th }}$ user is interfered by the transmission from other BSs to their respective users in the network. Pilot contamination is one of the causes of the intercell interference given that the same frequencies and the same set of pilots are reused throughout the network. Intracell interference caused by the use of nonorthogonal pilots within a cell, interference resulting from the lack of power control, or any other type of interference is not considered in this study.

The pilot reuse strategy has been considered as one of the solutions to mitigate the pilot contamination by setting apart the cells sharing the same pilots $[13,20]$. The pilot reuse strategy is designed in such a way that different orthogonal signals are assigned to each cell within the cluster. Here, the pilot reuse patterns of 1,3 , and 7 are investigated and their influence on the energy efficiency and power consumption has been assessed as well. The pilot reuse factor (1, 3, and 7 ) corresponds to the number of cells in a cluster that share the same set of orthogonal pilots. The higher the pilot reuse factor of a cluster, the less interference in the network since the cells belonging to different clusters (with the same set of orthogonal pilots) expected to interfere one another are far apart.

Under the conditions of the favorable propagation which require the propagation channels between the BS antennas array and the users to be mutually orthogonal, the received signal vector $\boldsymbol{q}_{\boldsymbol{l}}$ at the $K$ users in the $l^{\text {th }}$ cell can be expressed as a superposition of the signals intended to the users in $l^{\text {th }}$ cell and those from the neighboring BSs $[5,21]$ :

$$
\begin{aligned}
\boldsymbol{q}_{\boldsymbol{l k}}= & M \alpha_{M R T} \sqrt{p_{p}} \sqrt{p_{d l k}} \boldsymbol{D}_{\boldsymbol{l k l}} \boldsymbol{s}_{\boldsymbol{l} \boldsymbol{k}} \\
& +M \alpha_{M R T} \sqrt{p_{p}} \sum_{l^{\prime} \neq l}^{L} \sqrt{p_{d l^{\prime}}} \mathbf{D}_{\boldsymbol{l}^{\prime} l} \boldsymbol{s}_{\boldsymbol{l}^{\prime}}+\boldsymbol{n}_{\boldsymbol{d l}}
\end{aligned}
$$

where the first term of (2) refers to the signal received by the $k^{\text {th }}$ user in the $l^{\text {th }}$ serving cell, the second term refers to the contaminating signal (inter-cell interference) from the $l^{\prime t h}$ neighboring cell received by the $k^{\text {th }}$ user, and the third term refers to the additive white Gaussian noise (AWGN) whose elements are i.i.d with zero mean and variance $\sigma_{n}^{2}$.

Based on (2), the signal interference noise ratio for the $k^{\text {th }}$ user in the $l^{\text {th }}$ cell can be expressed in terms of variances of those different terms as follows:

$$
S I N R_{l k}=\frac{M \alpha_{M R T} p_{p} p_{d l k} \beta_{l k l}^{2}}{M \alpha_{M R T} p_{p} \sum_{j \neq l}^{L} p_{d j k} \beta_{j k l}^{2}+\sigma_{n}^{2}}
$$

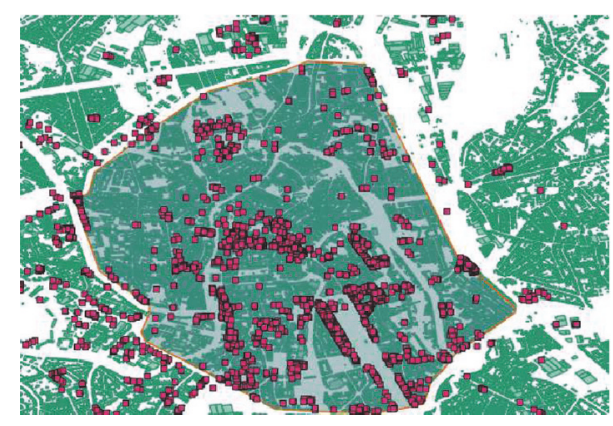

FIGURE 1: Selected area in Ghent, Belgium, and the possible locations of the base stations.

with $\alpha_{M R T}$ the normalization constant considered to satisfy the transmit power constraint at the BS [21]; $p_{p}$ is the power of the pilots (in W) and $p_{d l k}$ is the DL power of the serving base station (in W) in cell $l ; p_{d j k}$ is the DL power of the interfering BS (in W) in cell $j ; \beta_{j k l}$ represents the large-scale fading; and $\sigma_{n}^{2}$ is the noise variance. The achievable rate of the $k^{\text {th }}$ user in cell $l$ of a multicell system under imperfect channel state information (CSI) scales with the SINR as follows [19, 22]:

$$
\begin{aligned}
& R_{l k} \\
& \quad=\frac{\gamma^{D L}}{\eta}\left(1-\frac{\tau_{p}}{\tau_{c}}\right) \log _{2}\left(1+S I N R_{l k}\right)(\text { symbols } / s / H z)
\end{aligned}
$$

with $\eta$ the pilot reuse pattern, $S I N R_{l k}$ defined in (3), and $\gamma^{D L}$ is the data symbols portion (out of the coherence interval) reserved for DL transmission $\left(\gamma^{D L}=50 \%\right.$ [5]). The capacity that the massive MIMO BS can support is obtained after multiplying the sum capacity or spectral efficiency in (4) by the entire bandwidth of the channel:

$$
C_{l}=B \cdot \sum_{k=1}^{K} R_{l k}(\text { bits } / s)
$$

where $\mathrm{B}$ is the bandwidth of the channel (in $\mathrm{Hz}$ ), $R_{l k}$ is defined in (4), and $K$ is the number of users simultaneously served by the cell. The sum spectral efficiency in (5) does not grow indefinitely; the number of BS antennas $M$ and the length of the coherence interval $\tau_{c}$ are the limiting factors. So, the number of maximum $K$ users to be served simultaneously in a cell depends on these two factors. The authors in [12, 15] addressed that and set up a rule of thumb for the spectral efficiency which reaches its asymptotic limit when half of the coherence interval is spent on pilots. In other words, the spectral efficiency is maximized when $K=\tau_{p}=\tau_{c} / 2$.

\section{Method: Massive MIMO Network Design}

In this study, a realistic suburban area of $6.85 \mathrm{~km}^{2}$ in Ghent, Belgium (Figure 1), where a Belgian mobile operator has deployed its $4 \mathrm{G}$ network, has been used for the design of the massive MIMO network. The operator's network consists of 75 locations of the base stations to cover the entire area and provides voice and data services to the users. 
3.1. Problem Formulation. Given an initial set $S_{i}$ of $b$ massive MIMO 5G base stations, the optimization problem consists in determining a subset $S_{o p t}$ (from $S_{i}$ ) of optimal number of massive MIMO 5G base stations $b_{o p t}\left(b_{o p t}<b\right)$ that satisfies the constraints of minimizing the power consumption of the obtained network, responding to the data rates requested by the users and ensuring a coverage of at least $95 \%$ of the users:

$$
\begin{array}{cl}
\underset{p}{\operatorname{minimize}} & f(p) \\
\text { subject to } & b_{o p t} \leq b, \\
& \sum_{i} b r_{u} \leq C_{b s}, \\
& \operatorname{Cov} \geq 95 \%
\end{array}
$$

where $f(p)$ is a function of the power consumption of the designed network, $\sum_{i} b r_{u}$ is the sum of bit rate demanded by the users associated with a BS, depending on the applications used (video streaming, online gaming, etc.), $C_{b s}$ is the capacity of the BS, and Cov is the coverage of the users. This problem is solved by means of system-level simulations using the capacity-based network deployment tool $[23,24]$ that we describe further in this section. The analysis takes place at the busy hour $(\mathrm{BH})$ (worst case scenario), for which the operator supplied confidential data that showed that 224 simultaneous active users can be served by the $4 \mathrm{G}$ network. We assume the same number of simultaneous active users for the design of the massive MIMO network to ensure a fair comparison with the $4 \mathrm{G}$ network.

3.2. Massive MIMO System Setup and Scenarios. In this section, we present the main characteristics of the massive MIMO scenarios that we use for performance evaluation by means of system simulations. Moreover, we consider a Massive MIMO setup in which the operating frequency is set at $3.70 \mathrm{GHz}$ and the bandwidth at $20 \mathrm{MHz}$ (below $6 \mathrm{GHz}$ ). For realistic and practical reasons, the base stations use multiple antennas $(16,32,48,64$, and 256). The user equipment is assumed to have one single transmit antenna. The following scenarios have been considered $[14,25]$ :

(i) Scenario I (reference): 4G LTE network at $2.6 \mathrm{GHz}$, with $20 \mathrm{MHz}$ bandwidth without MIMO.

(ii) Scenario II: open exhibition environment (crowded) with services like live streaming requiring an average throughput per user of $20 \mathrm{Mbps}$. The coherence time is set to $2 \mathrm{~ms}$.

(iii) Scenario III: dense urban information society with application like real-time video gaming requiring an average throughput of $300 \mathrm{Mbps}$ per user. Here, the coherence time is set to $1 \mathrm{~ms}$.

Depending on the simulation scenario, the number of maximum simultaneous active users $K$ and the number of BSs deployed are generated automatically by the algorithms described in Figures 3 and 5. Referring to [5], the coherence time is set to $1 \mathrm{~ms}$ for scenarios with high user mobility (such as suburban dense areas) and to $2 \mathrm{~ms}$ for the crowded environment like stadium or open exhibition whereby users are almost static. The coherence bandwidth is set to $210 \mathrm{KHz}$. The effects of intercell interference are captured by means of a different pilot reuse pattern: 1,3 , and 7 . Based on the confidential data provided by a Belgian mobile operator, the number of simultaneous active users at peak hour in Ghent Centrum is derived and set to 224 .

3.3. Capacity-Based Network Deployment Tool. In this paper, a capacity-based network deployment tool is proposed for the design of optimal $5 \mathrm{G}$ networks. The tool is simulationbased and developed in java. The aim of the tool is to optimize the initial set $S_{i}$ of the base stations deployed in the area of interest based on the BS-user association principles with respect to the following optimization constraints: (i) the obtained network must be the least power consuming, (ii) it must respond to the instantaneous bit rates (voice and data) requested by the users, and (iii) it must provide a coverage to at least $95 \%$ of the users.

The flow chart of the tool describing all the processes is shown in Figure 2. First, two types of input files are required: (i) traffic files consisting of the list of users, their locations, and bit rates generated by means of the uniform distribution functions (explained further) and (ii) the additional files related to the link budget parameters, the 3-dimension (3D) shape of the environment, the power consumption parameters of the individual BS components, and the initial set of BSs locations. The second step consists of the network generation algorithm that simulates realistic networks based on the above input files. From the initial set of possible locations of the BSs which are assumed inactive, the algorithm estimates the path loss between each BS and the user; the BS with the lowest path loss and available capacity to support the user's demand in terms of data rate is enabled and associated with that user. During this step, the BS antenna assignment algorithm is invoked to control the input power of the BS since it influences the power consumption. So the users are assigned only the required number of BS antennas to be associated instead of accounting on the maximum transmit power of the BS. The output of the algorithm results into an optimal massive MIMO 5G network meeting the requirements in terms of the low-power consumption and the coverage of the users (at least 95\%).

For each considered scenario, we run multiple simulations (40 simulations in total) to ensure a good estimation of the relevant parameters investigated such as the number of BSs, the power consumption, the energy efficiency, the BS capacity, and the multiplexing gain. This latter refers to the number of users that can be served simultaneously by a base station during the coherence interval. The higher the multiplexing gain, the larger capacity the base station provides.

In the following lines, we discuss the different steps of the proposed tool with more details.

3.3.1. Step 1: Creation of the Traffic Files. Here, based on the confidential data provided by the mobile operator, we generate the files which contain the traffic information related to the maximum number of simultaneous active users, their 


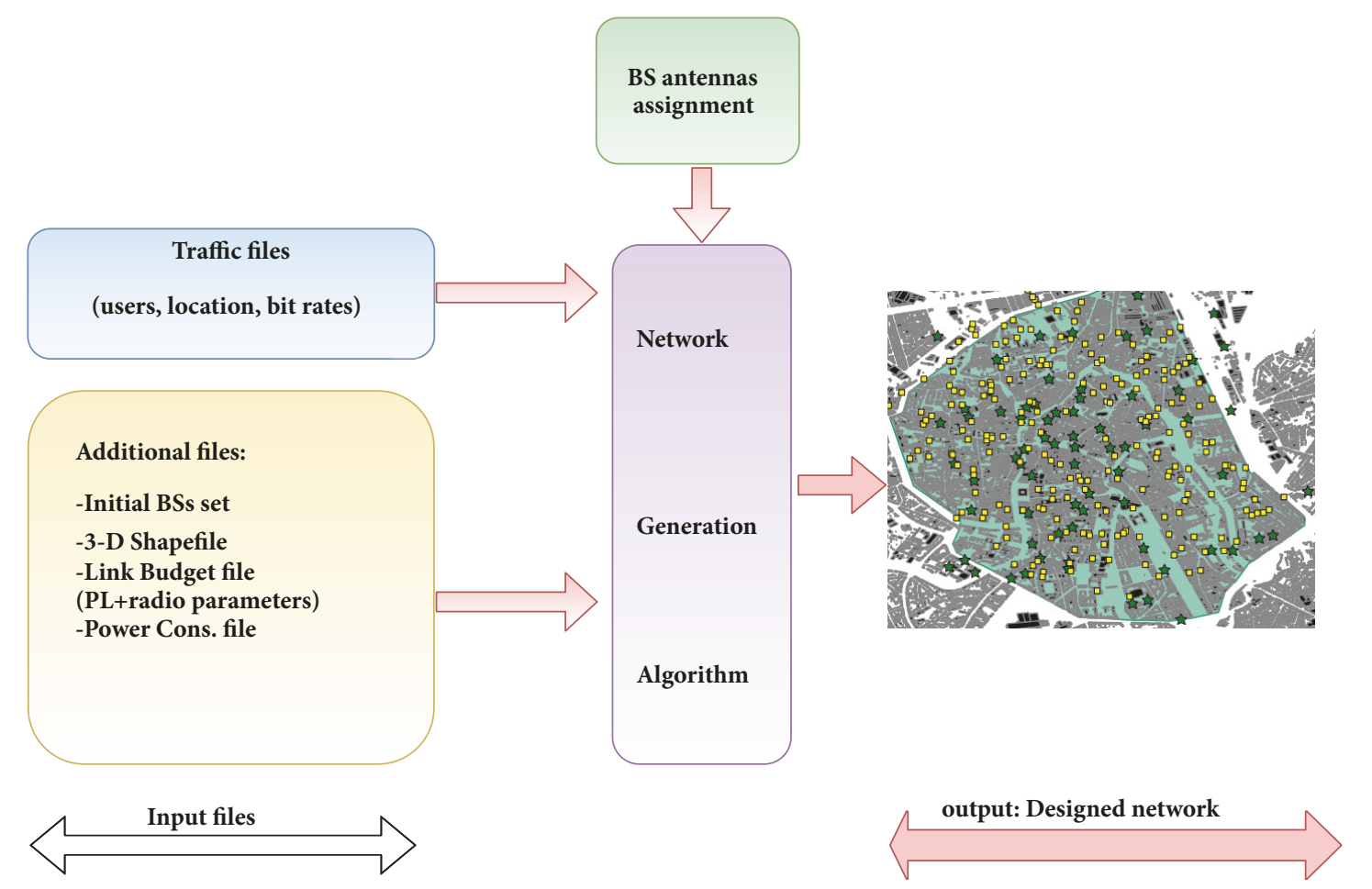

FIGURE 2: Flow chart of the network design process: yellow squares refer to the users while the green stars refer to the BSs.

locations, and their bit rates obtained thanks to the below distributions functions as shown in Figure 3. These traffic files will serve as inputs for the network generation algorithm.

(i) User distribution (Figure 3, step 1): this distribution returns the maximum number of simultaneous active users, based on the ratio between the daily data usage and the data usage at business hours. These are confidential data provided by a Belgian 4G mobile operator. To match with $5 \mathrm{G}$ applications requiring very high throughput such as live streaming and realtime gaming, these data rates have been extrapolated based on $38 \%$ yearly increase, as reported in the Ericsson forecast [26].

(ii) Location distribution (Figure 3, step 3): a uniform distribution is considered for the location of each user within the area of interest of the simulation. Each location will have the same chance to be chosen as a user location [24].

(iii) Bit rate distribution (Figure 3, step 3): this distribution returns the bit rate that the individual user demands for the service. A uniform bit rate distribution is assumed for the users since the coherence beamforming gain (that grows with $M$, the number of BS antennas) can help provide enough Signal to Noise Ratio (SNR) to enable higher bit rates for the users. The voice calls are made at $64 \mathrm{kbps}$ and the data transfer requested scales with the type of services offered in future $5 \mathrm{G}$ wireless network. These services are indicated in Section 3.2.
In total, 40 traffic files are generated since we run 40 simulations as explained above.

3.3.2. BS Antennas Assignment to the Users. This section describes the BS antenna assignment algorithm that accounts for the control of the BS transmit power and the accurate calculation of the coherence beamforming gain based on the effective number of BS antennas needed to associate a user, rather than considering all the $M \mathrm{BS}$ antennas. This latter case would lead to an overestimation of the beamforming gain to be used for the calculation of the maximum allowable path loss (MAPL). This algorithm is shown in Figure 4 and invoked by the network generation algorithm (Figure 5, step 4) to decide on the number of antennas $N(1 \leq N \leq M)$ needed by the user to connect to the serving BS.

For each user, the algorithm incrementally assigns $N$ antennas $(1 \leq N \leq M)$ to the user and each time uses the corresponding transmit power to evaluate the path loss (PL) the user is experiencing from the serving BS (Figure 4, step 2). If the path loss (PL) experienced is less than the MAPL, then the number of antennas assigned to the user is set to $N$ (Figure 4, step 4). Otherwise, $N$ is incremented (Figure 4, step 5) and the same verification applies until the $P L \leq M A P L$ condition is met. If all antennas of a BS are used and the path loss condition is not met, then the algorithm moves to another BS for the same process (Figure 4, step 6). If all BSs are considered and none of them satisfies the path loss condition, then the user cannot be served (Figure 4, step 6). The same operations are repeated for all the users. 


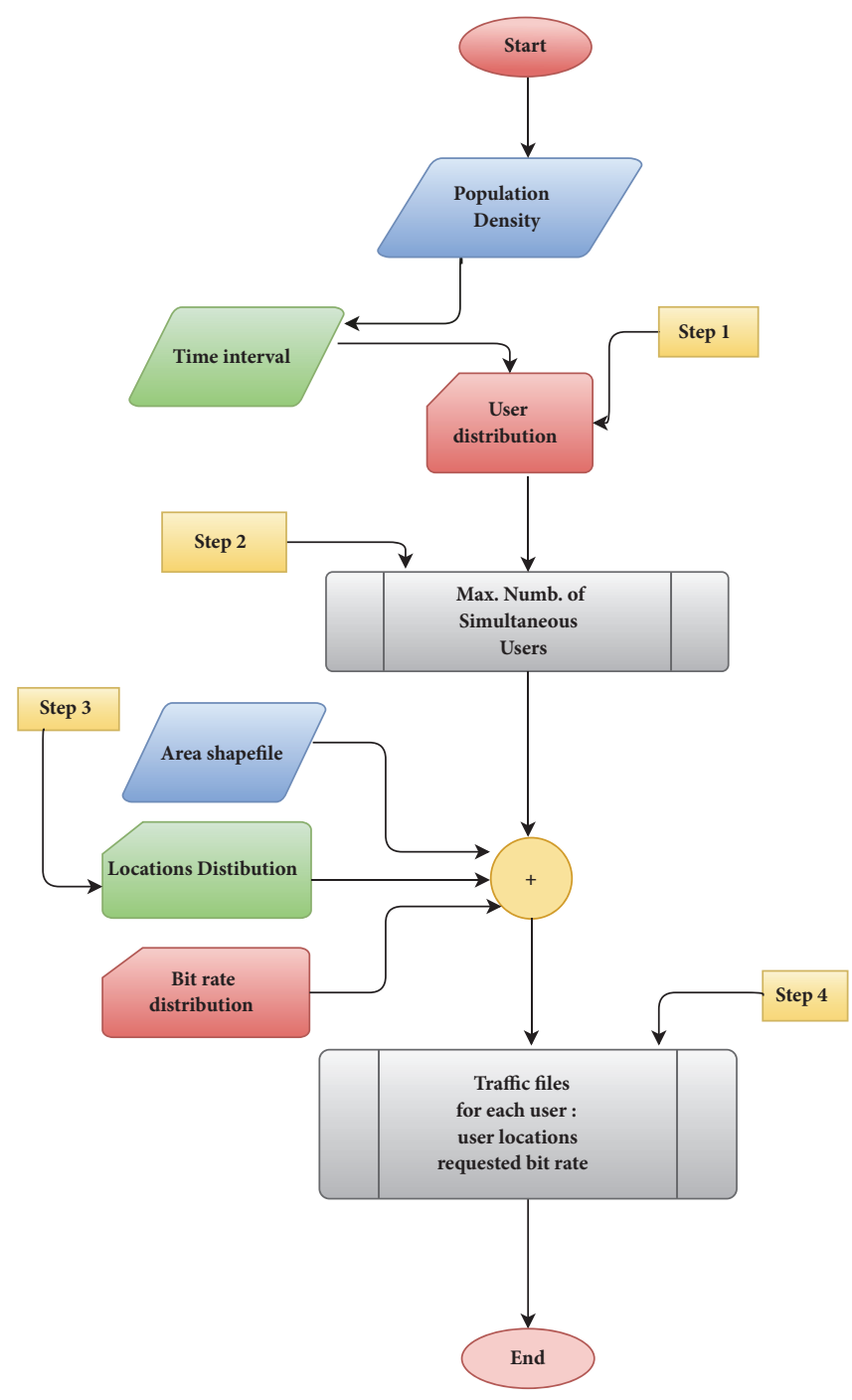

Figure 3: Diagram of the creation of traffic files.

3.3.3. Generation of Network Traffic. This capacity-based network deployment tool is based on the design of [23], where a 5G network was optimized with beamforming capability only. Here, in addition to beamforming, we implement the optimization algorithm to cope with the massive MIMO requirements in terms of the higher data rates imposed by the $5 \mathrm{G}$ applications, the sum spectral efficiency described in (5), the path loss, and the power consumption models. We consider both the 3GPP LTE Rel. 8 path loss model and the power consumption model proposed in [14] as they are well suited for massive MIMO. These models are detailed further in this section.

In this section, we describe the algorithm that generates the different optimal $5 \mathrm{G}$ networks as a solution to the optimization problem described earlier. In addition to the traffic files generated above, the following is needed to create the $5 \mathrm{G}$ network: a file with the set of possible locations of the base stations in the considered area, two geographic information system (GIS) shapefiles (one describing the environment and the second the digital terrain model depicting the building locations and heights within the environment), a file with the link budget parameters, and finally a file containing the power consumption values of the different components of the base station. This BS-user association algorithm ensures at least $95 \%$ of the users are served while optimizing the number of base stations to be deployed towards the power consumption. For each time interval of 1 hour, 40 simulations are done whereby 40 networks are created.

We assume all the BSs in the initial set of base stations are inactive. The aim of the algorithm is to activate the least possible BSs from this set whenever the conditions to associate a user to a BS are met and to ensure the power consumption of the entire network is minimized. This results into a subset of active BSs which will constitute the desired $5 \mathrm{G}$ networks.

For a new user in the area (Figure 5, step 3) to be associated with a BS, the path loss experienced and the requested bit rate must be lower than the MAPL and the effective capacity of the BS, respectively. This latter refers to the difference between the maximum capacity of the BS $C_{\max }$ 


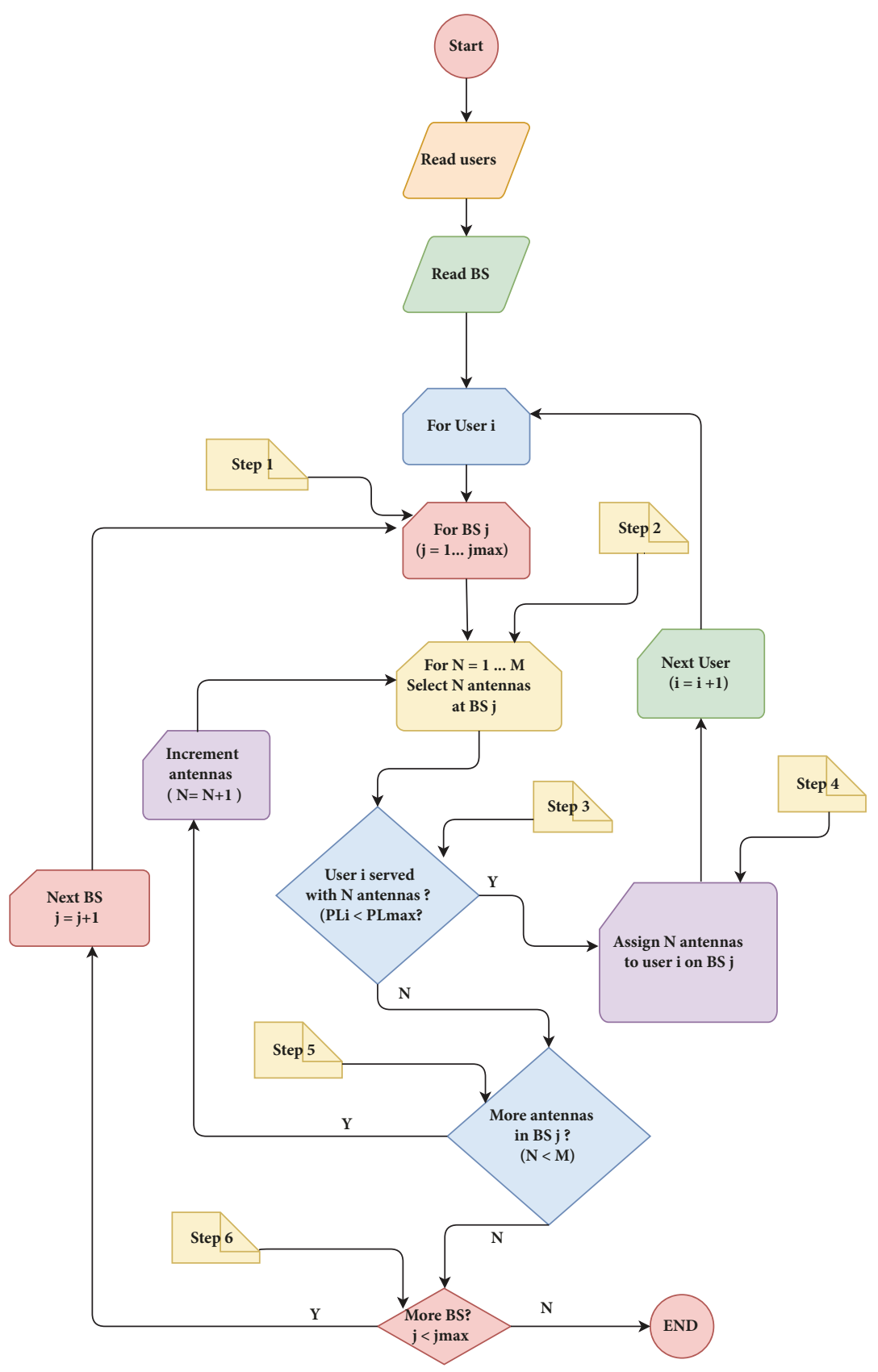

FIGURE 4: BS antenna assignment algorithm.

in (5) and the sum of the bit rates of the already connected users. During the evaluation of the path loss, not all the $M$ BS antennas are needed for the coherent beamforming gain. The algorithm in Figure 4 is invoked (Figure 5, step 4) and assigns the necessary number of BS antennas required for the user to receive enough signal strength from the BS. This also contributes to the reduction of the power consumption of the BS as some RF chains might not be solicited. The algorithm will check if it is possible to connect the user to an existing active BS. If it is not possible, the power of the existing active BS is incremented and the association conditions are evaluated again. If it is still not possible to connect the user to an already existing active BS, a new base station will be enabled (activated) on condition that the path loss between this base station and the user is the lowest among the disabled base stations (Figure 5, step 5). At the same time, the requirements related to the effective capacity of the newly enabled BS should be met as well to support the applications or the services requested by the user.

Moreover, in Figure 5, step 6, the algorithm will assess the possibility of migrating the users already connected to other active base stations to this newly enabled base station, since they may experience a lower path loss from this new base station. If this is the case, then there is a possibility to 


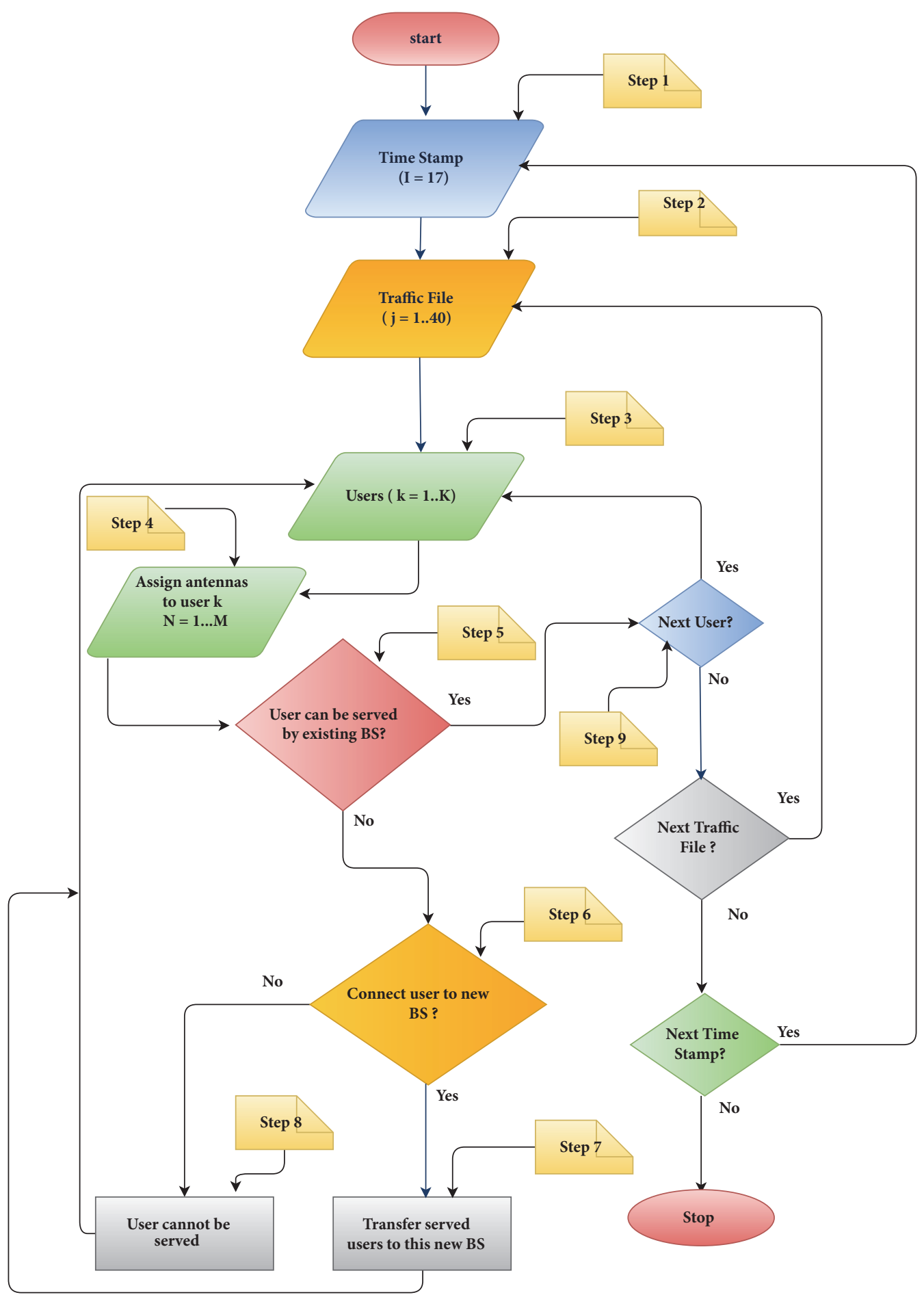

FIGURE 5: Network generation algorithm.

reduce the input power of the base station the user is removed from. When all users are migrated to a newly enabled $\mathrm{BS}$, the existing active BS is left empty and is deactivated automatically. If no base station can be enabled or all base stations are already active, the user cannot be served. This operation is repeated for all the users defined in the traffic file for a given time interval. The new obtained subsets of BSs with their respective placements within the area of interest are then optimal. The number of users associated with this subset of active BSs is aggregated and compared to the 224 users to ensure that at least $95 \%$ of them are covered.

While the link budget parameters used for the computation of this algorithm are given in Table 1, the path loss model 
TABLE 1: Massive MIMO common link budget parameters [14].

\begin{tabular}{lc}
\hline Parameters & Values \\
\hline Carrier frequency & $3.7 \mathrm{GHz}$ \\
Channel bandwidth & $20 \mathrm{MHz}$ \\
Transmit antenna element gain & $10 \mathrm{dBi}$ \\
Transmit array antenna feed loss & $3 \mathrm{~dB}$ \\
Base station total radiated power & $43 \mathrm{dBm}$ \\
Number of MS antenna elements & 1 \\
MS transmit power & $23 \mathrm{dBm}$ \\
Receive antenna element gain & $0 \mathrm{dBi}$ \\
SNR & $(7.39,15.4,17.5) \mathrm{dB}{ }^{1}$ \\
Path loss exponent & 3.8 \\
Coherence bandwidth & $210 \mathrm{kHz}$ \\
Implementation loss & $3 \mathrm{~dB}$ \\
RX noise figure & $7 \mathrm{~dB}$ \\
Other losses (shadow, fading) & $20 \mathrm{~dB}$ \\
\hline
\end{tabular}

${ }^{1}$ Values of signal-to-noise ratio corresponding to [1/2 BPSK, 1/2 QPSK, 1/2 16-QAM], [15].

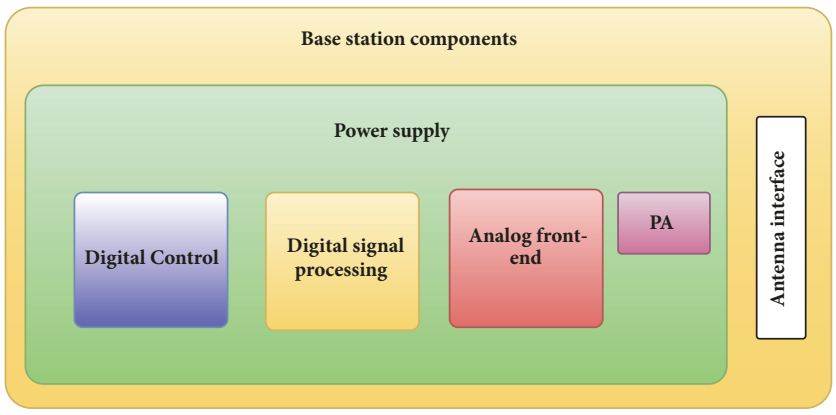

FIGURE 6: Massive MIMO base station power model components.

and the power consumption model considered are discussed below:

(i) Path Loss Model.

The path loss model has been obtained from the $3 \mathrm{GPP}$ LTE Rel. 8 as proposed in [14] for a fair comparison with the $4 \mathrm{G}$ network:

$$
\text { Pathloss }=P L(d)+\log F
$$

with

$$
\begin{aligned}
P L(d)= & 40\left(1-4 \cdot 10^{-3} \cdot h_{b}\right) \log _{10}(d)-18 \log _{10}\left(h_{b}\right) \\
& +21 \log _{10}(f)+80 d B
\end{aligned}
$$

where $d$ is the distance in $\mathrm{m}$ between the BS and the user; $f$ is the carrier frequency in $\mathrm{MHz} ; h_{b}$ is the antenna height of the BS in $m$; and $\log F$ is a $\log$ normally distributed large-scale fading variable with standard deviation of $10 \mathrm{~dB}$.

(ii) Massive MIMO Power Consumption Model.

The main components or subcomponents of the massive MIMO base station and their respective power consumption models are discussed. In this analysis, we consider the power consumption model approach of [27]; this latter has been complemented with the scaling rules in $[14,28]$. This choice is motivated by the fact that it provides more details about the dedicated components specific to massive MIMO. In addition, it sets up a rule-of-thumb that governs the way the power consumption varies with the parameters like the bandwidth, the frequency, the quantization resolution, and so forth. Based on this approach, a typical massive MIMO base station architecture is shown in Figure 6 and consists of the following components:

(a) Power amplifier (PA): thanks to its low perantenna power output, massive MIMO base stations do not require an external power amplifier. A predriver (last buffer stage of the analog frontend) is used instead [28]. This latter can generate the required output power of the antenna element. The power consumption of the predriver is modeled in the same way as a traditional PA, provided that it delivers an output power level with an efficiency set to a maximum of 50\% [14]. 
TABLE 2: Reference power consumption of analog front-ends.

\begin{tabular}{lcc}
\hline Subcomponent & $\begin{array}{c}\text { Downlink } \\
{[\mathbf{m W}]}\end{array}$ & $\begin{array}{c}\text { Uplink } \\
{[\mathbf{m W}]}\end{array}$ \\
\hline Predriver & 115 & 0 \\
Modulator & 200 & 0 \\
Frequency synthesis & 125 & 125 \\
Clock generation & 75 & 75 \\
DAC & 225 & 0 \\
LNA & 0 & 125 \\
Mixer & 0 & 200 \\
VGA & 0 & 63 \\
ADC & 0 & 175 \\
\hline
\end{tabular}

TABLE 3: Scaling exponents for analog front-ends subcomponents.

\begin{tabular}{|c|c|c|c|c|c|c|}
\hline Subcomponent & $\mathrm{BW}^{2}$ & S.E. ${ }^{2}$ & Ant. $^{2}$ & Load & Streaming & $\mathrm{Q}^{2}$ \\
\hline Predriver & 1 & 0 & 1 & 0 & 0 & 0 \\
\hline Modulator & 1 & 0 & 1 & 0 & 0 & 0 \\
\hline Freq. synthesis & 0 & 0 & 1 & 0 & 0 & 0 \\
\hline Clock generation & 0 & 0 & 0.5 & 0 & 0 & 0 \\
\hline DAC & 1 & 0 & 1 & 0 & 0 & 1 \\
\hline LNA & 1 & 0 & 1 & 0 & 0 & 0 \\
\hline Mixer & 1 & 0 & 1 & 0 & 0 & 0 \\
\hline VGA & 1 & 0 & 1 & 0 & 0 & 0 \\
\hline $\mathrm{ADC}$ & 1 & 0 & 1 & 0 & 0 & 1 \\
\hline
\end{tabular}

${ }^{2}$ BW: bandwidth; S.E.: spectral efficiency; Ant.: antenna; and Q: quantization resolution.

(b) Analog front-end: this component consists of relevant subcomponents usable in UL, DL, or both. The frequency synthesizer and the clock generation are used in both UL and DL. Predrivers, modulators, and digital-to-analog converters (DACs) are only used in the DL, while low-noise amplifiers (LNAs), mixers, variable-gain amplifiers (VGAs), and analog-todigital converters (ADCs) are only active in UL. Tables 2 and 3 summarize the power consumption of these subcomponents for a reference scenario of $20 \mathrm{MHz}$, a single antenna, and 24-bit quantization [28]. The power model of the analog front-end scales with three different design parameters: the bandwidth, the number of BS antennas $M$, and the digital quantization resolution $\mathrm{Q}$. The following equation provides the power consumption model of the analog front-end, valid for UL and DL $[14,28]$ :

$P_{\text {analog }}=\sum_{i \in I_{\text {analog }}} P_{i, r e f} \prod_{x \in \chi_{\text {analog }}}\left(\frac{x_{\text {act }}}{x_{\text {ref }}}\right)^{s_{i, x}}$

where $I_{\text {analog }}$ is the set of analog front-end subcomponents (predrivers, modulators, etc.); $\chi_{\text {analog }}$ is the set of scenario parameters $x$ (bandwidth, antennas, and quantization resolution);
$P_{i, r e f}$ is the power consumption of analog frontend subcomponents for the reference scenario parameters $x_{r e f}(20 \mathrm{MHz}$, single antenna, 24bit quantization); $x_{a c t}$ is the scaling parameter $x$ at a given operating point; and $s_{i, x}$ is the scaling exponent of each analog front-end subcomponent with regard to any of the parameter $x$.

(c) Digital signal processing or digital baseband: it consists of the following subcomponents: filtering, sampling, OFDM modulation/demodulation, channel coding, precoding/combining, and so forth. The power consumption of these subcomponents is modeled in a similar way to that of the analog subcomponents but scales with more additional scenario parameters. The digital power consumption is modeled through the digital complexity in terms of Gigaoperations per second (GOPS); these values are then translated into power consumption values as a function of the intrinsic efficiency of the hardware, measured in GOPS/W. The reference conversion factor is set to $8 \mathrm{GOPS} / \mathrm{W}$ [28]. The digital baseband power consumption depends on the system load $\gamma$ (in the frequency-domain), the number of users $K$, the data streams, and the spectral efficiency SE assigned to each user. 
TABLE 4: Reference power consumption of digital components.

\begin{tabular}{lccc}
\hline Subcomponent & $\begin{array}{c}\text { Downlink } \\
\text { [GOPS] }\end{array}$ & $\begin{array}{c}\text { Uplink } \\
\text { [GOPS] }\end{array}$ & $\begin{array}{c}\text { Training } \\
\text { [GOPS] }\end{array}$ \\
\hline Filtering & 6.7 & 6.7 & 6.7 \\
Up/Downsampling & 2 & 2 & 2 \\
FFT/IFFT & 0.5 & 0.5 & 0.5 \\
MIMO precoding & 0.04 & 0.04 & 0 \\
Synchronization & 0 & 2 & 0 \\
Channel estimation & 0 & 0 & 0.01 \\
OFDM Mod/Demod & 1.3 & 2.7 & 2.7 \\
Mapping/Demapping & 1.3 & 2.7 & 2.7 \\
Channel coding & 1.3 & 8 & 0 \\
Control & 2.47 & 1 & 1 \\
Network & 8 & 5.3 & 0 \\
\hline
\end{tabular}

TABLE 5: Scaling exponents for digital subcomponents.

\begin{tabular}{|c|c|c|c|c|c|c|}
\hline Subcomponent & BW & S.E. & Ant. & Load & Streaming & $\mathbf{Q}$ \\
\hline Filtering & 1 & 0 & 1 & 0 & 0 & 1.2 \\
\hline Up/Downsampling & 1 & 0 & 1 & 0 & 0 & 1.2 \\
\hline FFT/IFFT & 1.2 & 0 & 1 & 0 & 0 & 1.2 \\
\hline MIMO precoding & 1 & 0 & 1 & 1 & 1 & 1.2 \\
\hline Synchronization & 0 & 0 & 1 & 0 & 0 & 1.2 \\
\hline Channel estimation & 1 & 0 & 1 & 0.5 & 1 & 1.2 \\
\hline OFDM Mod/Demod & 1 & 0 & 1 & 0.5 & 0 & 1.2 \\
\hline Mapping/Demapping & 1 & 1.5 & 0 & 1 & 1 & 1.2 \\
\hline Channel coding & 1 & 1 & 0 & 1 & 1 & 1.2 \\
\hline Control & 0 & 0 & 0.5 & 0 & 0.2 & 0.2 \\
\hline Network & 1 & 1 & 0 & 1 & 0 & 0 \\
\hline
\end{tabular}

This model is given by the following equation $[14,28]$ :

$$
P_{\text {baseband }}=\sum_{i \in I_{\text {bassband }}} P_{i, r e f} \prod_{x \in \chi_{\text {basebeand }}}\left(\frac{x_{a c t}}{x_{r e f}}\right)^{s_{i, x}}
$$

where $I_{\text {baseband }}$ is the set of the digital subcomponents; $\chi_{\text {baseband }}$ is the set of scenario parameters $x$ (bandwidth, spectral efficiency, antennas, load factor, number of users, streams, and quantization resolution); $x_{r e f}$ is a reference value; $x_{a c t}$ is the operation value with regard to the scenario parameter $x$; and $s_{i, x}$ is the scaling exponent. Tables 4 and 5 summarize the power consumption and the scaling exponents of the digital baseband subcomponents, respectively, for a reference scenario of $20 \mathrm{MHz}$, a single antenna, and a 24-bit quantization.

Besides the digital signal processing, some additional digital functions are required: platform control (data flow management, etc.), network processing (higher-layer protocols), and backhauling to the core network. They are modeled together with the digital baseband components and indicated in Tables 4 and 5 .

(d) Power systems $\left(P_{s y s}\right)$ : the power system mainly includes the AC/DC and DC/DC converters as well as the cooling system. Both the AC/DC and DC/DC are simply modeled as having $8 \%$ losses each; i.e., $92 \%$ of their power consumption gets transferred to the other components in the system.

Based on the above equations, the total power consumption of the massive MIMO BS can be modeled as follows:

$$
P_{\text {total }}=P_{\text {analog }}+P_{\text {baseband }}+P_{\text {sys }}
$$

3.4. Energy Efficiency. Deciding which designed network provides the better energy efficiency is not simple since various parameters are taken into account (covered area, capacity, covered users, etc.). For a better comparison, we consider an energy efficiency (EE) metric that combines multiple network performance parameters such as the covered area, the ergodic achievable bit rate, and the coverage of the users. The energy 
TABLE 6: Open exhibition: simulation results (95 percentile).

\begin{tabular}{|c|c|c|c|c|c|c|}
\hline Pilot reuse pattern & MIMO Config & $\begin{array}{c}\text { \# BS } \\
{[-]}\end{array}$ & $\begin{array}{l}\text { P.C. } \\
{[\mathrm{kW}]}\end{array}$ & $\begin{array}{c}\text { BS Capacity } \\
\text { [Mbps] }\end{array}$ & $\begin{array}{c}\text { Energy } \\
\text { Efficiency } \\
{\left[\mathrm{km}^{2} \cdot M b p s / W\right]}\end{array}$ & $\begin{array}{c}\text { Multiplexing } \\
\text { gain }\end{array}$ \\
\hline & 4G LTE & 33 & 46.50 & 449.48 & 14.57 & - \\
\hline \multirow{5}{*}{1} & $16 x 1$ & 35 & 1.34 & 25451.17 & 3794.61 & 6.2 \\
\hline & $32 \times 1$ & 29 & 1.37 & 21199.36 & 4197.21 & 7.66 \\
\hline & $64 \times 1$ & 22 & 1.43 & 16047.67 & 4196.41 & 10.18 \\
\hline & $128 \mathrm{x} 1$ & 17 & 1.71 & 12411.79 & 3575.03 & 13.18 \\
\hline & $256 \times 1$ & 13 & 2.24 & 9497.50 & 2789.83 & 17.23 \\
\hline \multirow{5}{*}{3} & $16 x 1$ & 35 & 1.34 & 96227.31 & 3755.19 & 6.13 \\
\hline & $32 \times 1$ & 29 & 1.37 & 81049.58 & 4186.20 & 7.69 \\
\hline & $64 \times 1$ & 22 & 1.43 & 62368.93 & 4208.08 & 10.18 \\
\hline & $128 \mathrm{xl}$ & 16 & 1.61 & 45835.20 & 3777.02 & 14.00 \\
\hline & $256 \times 1$ & 12 & 2.08 & 34926.36 & 2980.73 & 18.51 \\
\hline \multirow{5}{*}{7} & $16 x 1$ & 35 & 1.34 & 57994.01 & 3764.66 & 6.17 \\
\hline & $32 \times 1$ & 28 & 1.33 & 49624.49 & 4342.37 & 7.94 \\
\hline & $64 \times 1$ & 22 & 1.43 & 41114.07 & 4257.15 & 10.18 \\
\hline & $128 \mathrm{x} 1$ & 17 & 1.72 & 33582.19 & 3567.66 & 13.1 \\
\hline & $256 \times 1$ & 13 & 2.24 & 26645.74 & 2771.68 & 17.23 \\
\hline
\end{tabular}

TABLE 7: Information society: simulation results (95 percentile).

\begin{tabular}{|c|c|c|c|c|c|c|}
\hline Pilot reuse pattern & MIMO Config & $\begin{array}{r}\# \mathrm{BS} \\
{[-]}\end{array}$ & $\begin{array}{l}\mathrm{PC} \\
{[\mathrm{kW}]}\end{array}$ & $\begin{array}{c}\text { BS Capacity } \\
{[\mathrm{Mbps}]}\end{array}$ & $\begin{array}{r}\text { Energy Efficiency } \\
{\left[\mathrm{km}^{2} \cdot \mathrm{Mbps} / W\right]}\end{array}$ & $\begin{array}{c}\text { Multiplexing } \\
\text { gain }\end{array}$ \\
\hline & 4G LTE & 33 & 46.50 & 449.48 & 14.57 & - \\
\hline \multirow{5}{*}{3} & $16 x 1$ & 39 & 1.49 & 79781.79 & 63288.86 & 5.74 \\
\hline & $32 \mathrm{x} 1$ & 38 & 1.79 & 78481.91 & 52624.40 & 5.89 \\
\hline & $64 \times 1$ & 37 & 2.41 & 76943.04 & 39194.20 & 6.05 \\
\hline & $128 x 1$ & 36 & 3.63 & 75232.26 & 26010.16 & 6.22 \\
\hline & $256 \times 1$ & 36 & 6.20 & 75496.39 & 15230.01 & 6.22 \\
\hline \multirow{5}{*}{7} & $16 \times 1$ & 45 & 1.72 & 74563.73 & 54855.52 & 4.98 \\
\hline & $32 \times 1$ & 44 & 2.08 & 77703.82 & 45448.34 & 5.09 \\
\hline & $64 \times 1$ & 37 & 2.41 & 69146.39 & 39194.20 & 6.05 \\
\hline & $128 \mathrm{x} 1$ & 36 & 3.64 & 70895.74 & 25938.11 & 6.20 \\
\hline & $256 x 1$ & 36 & 6.20 & 73788.19 & 15230.01 & 6.22 \\
\hline
\end{tabular}

efficiency (EE) metric in this study is defined by the following equation [24] in $\left[\mathrm{km}^{2} \cdot \mathrm{Mbps} / \mathrm{W}\right]$ :

$$
E E=\frac{A \cdot U \cdot \sum_{j=1}^{n} B_{j}}{\sum_{j=1}^{n} P_{e l j}}
$$

where $A$ is the area covered by the designed network (in $\mathrm{km}^{2}$ ), $U$ is the number of served users, $\sum_{j=1}^{n} B_{j}$ is the total achievable bit rate offered by the network (in $M b p s$ ) obtained with (5), $\sum_{j=1}^{n} P_{e l j}$ is the total power consumption of the network (in Watt) obtained with (11), and $n$ is the total number of active BSs of the designed network. The higher the EE value is, the more energy-efficient the network is.

We would like to mention that other EE metrics could be used, which would result into different values. Here, we consider this metric that merges the effects of individual existing metrics $A / \sum P_{e l}$ [29], $U / \sum P_{e l}$ [30], and $\sum B / \sum P_{e l}$ [31] corresponding to the power consumed by the network to cover a given geographical area, to serve the users and to deliver the required achievable bit rates, respectively. Since the designed networks obtained from the simulations differ from one scenario to another in terms of geographical area covered, number of users served, and total achievable bit rates, such a combined metric will be useful to allow a fair comparison between the scenarios.

\section{Results and Discussions}

In this section, we discuss the performance of the different $5 \mathrm{G}$ massive MIMO networks obtained thanks to the system level simulations, based on the scenarios described in Section 3. Tables 6 and 7 show the results of the simulations. These are 

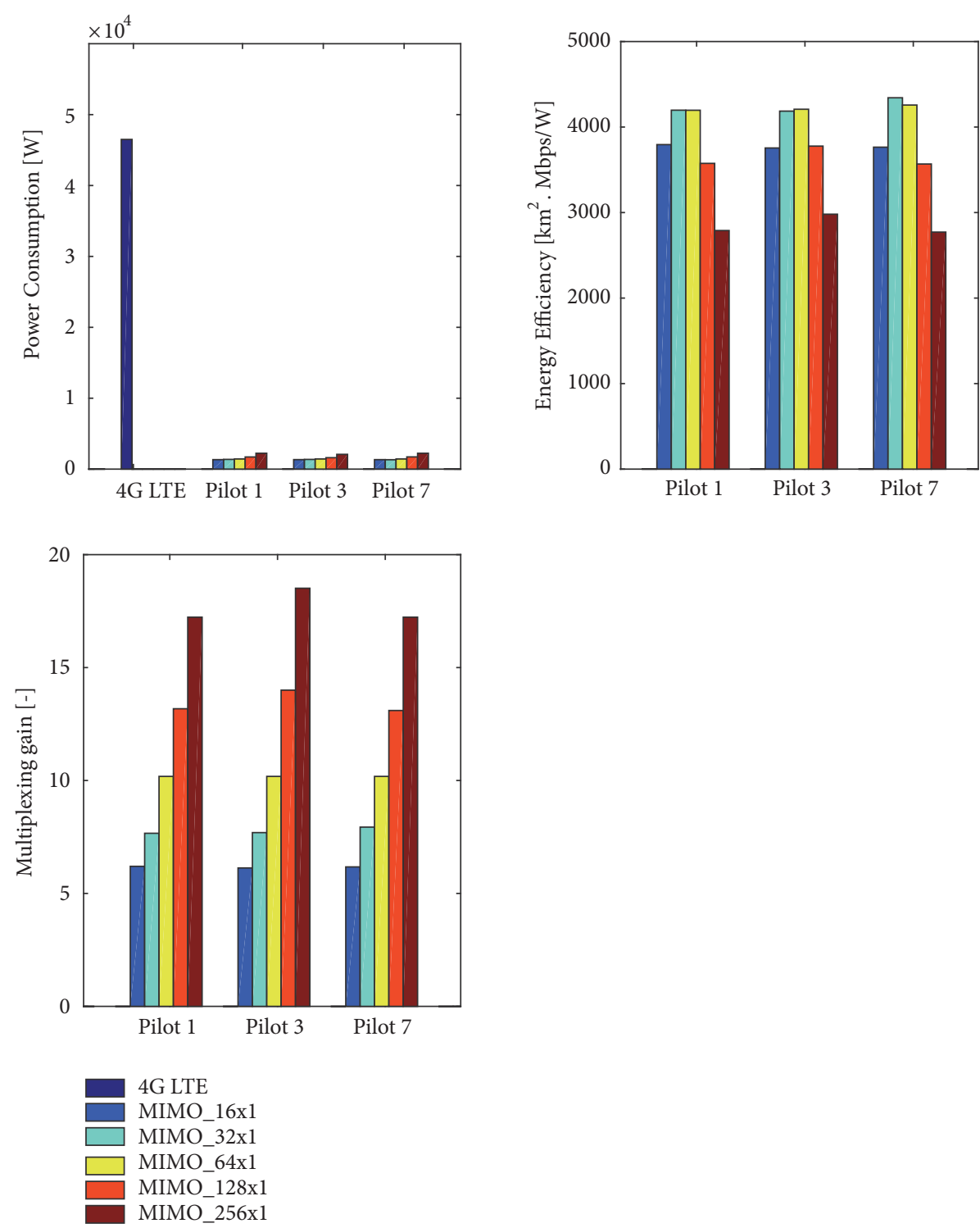

FIGURE 7: Comparison of different parameters for open exhibition scenario: total power consumption of the network, energy efficiency, and multiplexing gain. Pilots 1, 3, and 7 refer to pilot reuse patterns 1, 3, and 7, respectively.

analyzed later and compared with those related to the $4 \mathrm{G}$ reference network.

In all the scenarios investigated, Tables 6 and 7 show that a massive MIMO BS consumes 8 times less power than the $4 \mathrm{G}$ reference scenario and provides almost 200 times more capacity. In fact, based on the results, a massive MIMO BS consumes only $172 \mathrm{~W}$, while the $4 \mathrm{G}$ base station needs 1.4 $\mathrm{KW}$ to provide services to the users. This is explained by the low-power consumption components used in massive MIMO base stations, with simpler architecture. Moreover, the large number of antennas at the massive MIMO BS implies low output power level at each antenna element. Therefore, there is no need to keep the traditional external power amplifier as in 4G; a predriver at the buffer stage of the front-ends is used instead, to comply with this low output power level requirement. However, Figures 7 and 8 show that the power consumption of the massive MIMO networks increases with the number of BS antennas. In scenario II (Figure 7), when the number of antennas increases from 16 to 256 , the power consumption increases by $67 \%$, while, in scenario III (Figure 8), the increase is more pronounced. When 256 antennas are used at the BS side, the power consumption is 4 times higher compared to the case where 16 antennas are used. This is mostly attributed to the number of $\mathrm{RF}$ chains behind each antenna. There are as many antennas as the number of associated RF chains. But this increase is still lower than the power consumption of the $4 \mathrm{G}$ reference network.

Based on the chosen energy efficiency metric considered, Figures 7 and 8 show a degradation of the energy efficiency as the circuit power scales with the number of massive MIMO BS antennas. The more the antennas are, the less energy efficient the massive MIMO network is because of the power consumption that increases with the number of 

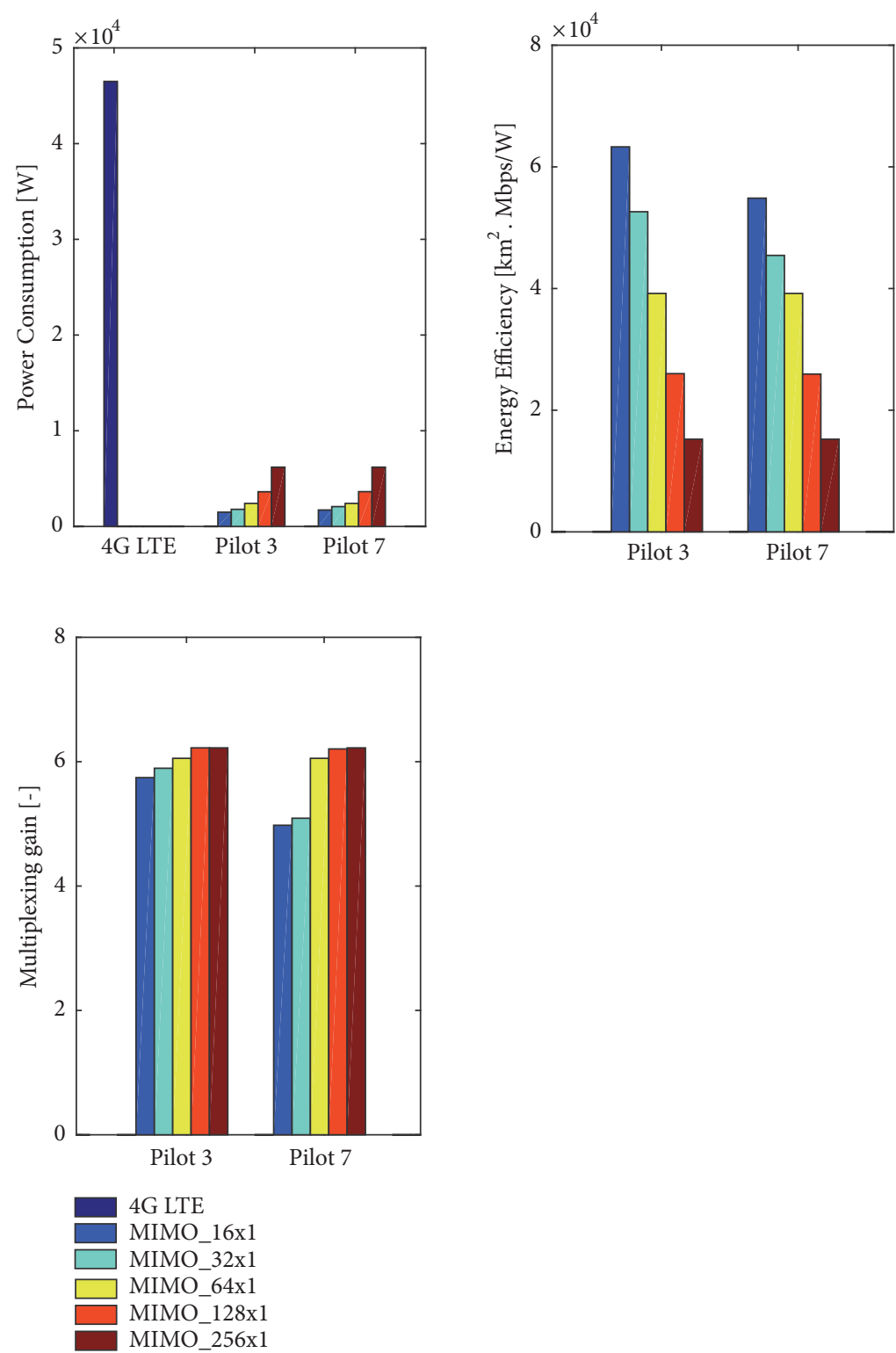

FiguRE 8: Comparison of different parameters for urban information society scenario: total power consumption of the network, energy efficiency, and multiplexing gain.

BS antennas. However, massive MIMO networks present impressive performances in terms of energy efficiency in comparison with the $4 \mathrm{G}$ reference network. They are 400 times more energy efficient than $4 \mathrm{G}$ and this performance is achieved thanks to the number of simultaneous users that can be multiplexed contributing to a higher throughput of the networks and the low-power consumption equipment they are using.

Another interesting result of the massive MIMO network is the multiplexing gain, i.e., the number of simultaneous users that can be multiplexed in the same time-frequency resource (coherence interval). Figure 9 shows that the multiplexing gain is important when the number of antennas at the base stations increases, despite the effect of the intercell interference (pilot contamination). Even in the most interfering situation where the pilot reuse factor is set to 1 , the strength of the spatial multiplexing gain is still noticeable through the increase of the served users. In scenario II (open exhibition), the multiplexing gain is multiplied by 3 when the number of antennas grows from 16 to 256, while, in scenario III (Dense urban information society), the multiplexing gain is increased by $25 \%$ when the number of antennas varies from 16 to 256 (Figure 9).

However, when comparing the two scenarios, there are 3 times more users served by multiplexing in scenario II than in scenario III (Tables 6 and 7). This is mainly explained by the mobility of the users: in an environment with moderate mobility of the users like an open exhibition, it is required to use a longer coherence interval ( $2 \mathrm{~ms}$ in our study) that provides more room for longer pilots (210 pilots in our study) 


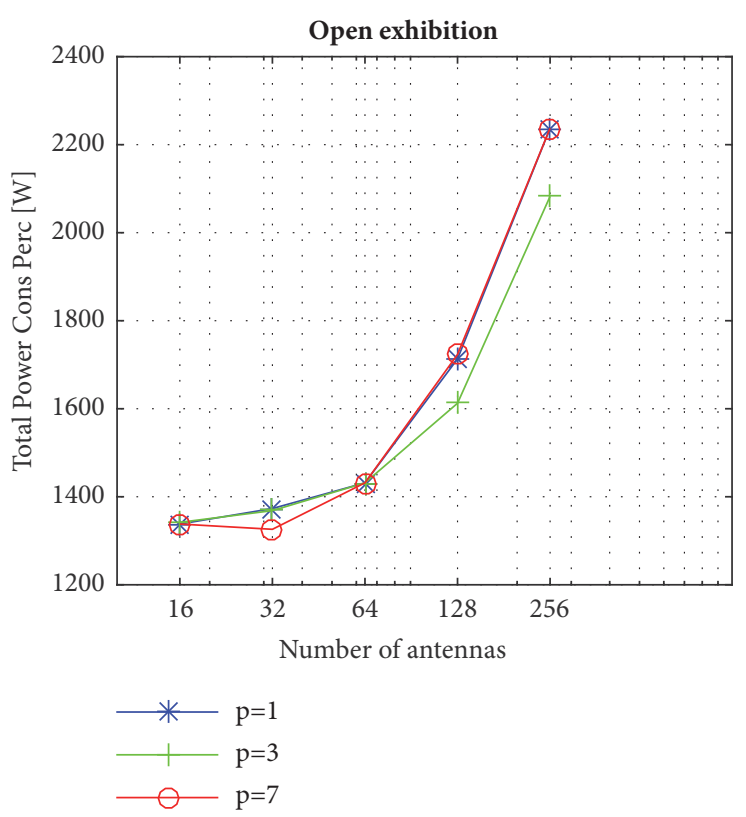

(a)

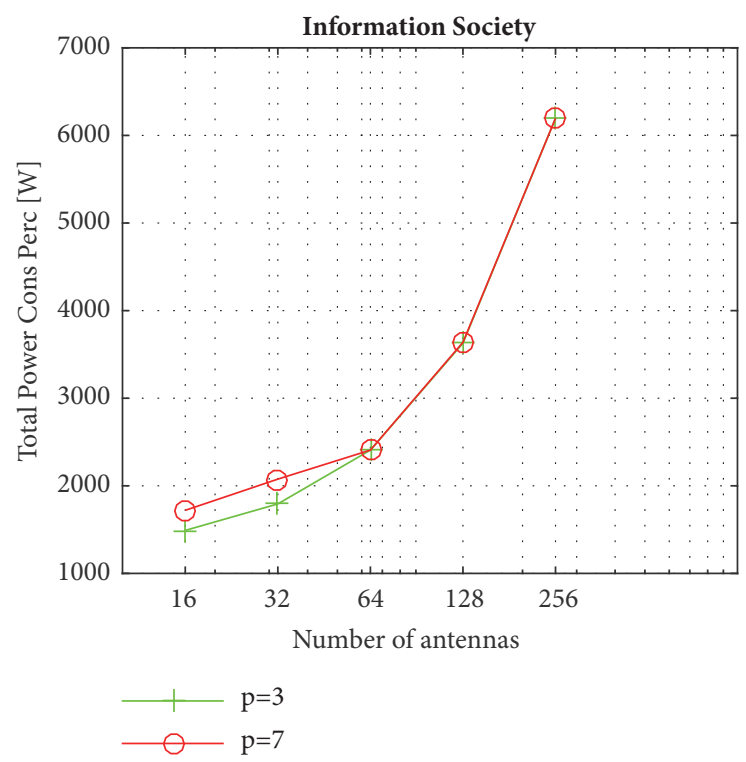

(c)

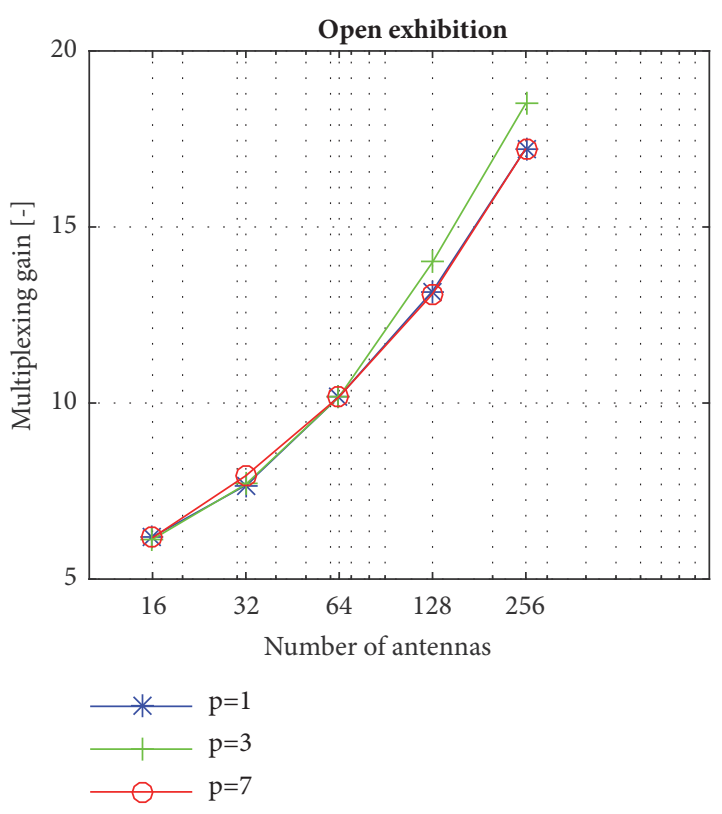

(b)

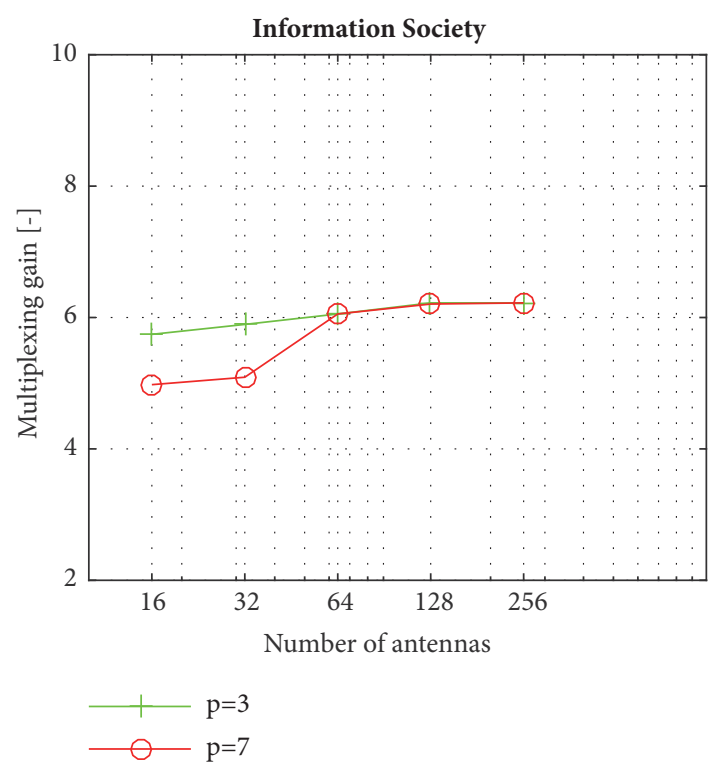

(d)

FIGURE 9: Effects of pilot contamination and number of BS antennas.

and to give possibilities to serve many users, while the high mobility environment requires a shorter coherence interval (1 $\mathrm{ms}$ in our study). The massive MIMO network (256xl) in the open exhibition scenario requires 2 times less base stations and therefore consumes almost 3 times less power than the networks (256xl) in the suburban information society (Tables 6 and 7$)$.

In terms of base station capacity, it is shown in Tables 6 and 7 that the pilot reuse pattern of 3 shows better results compared to the pattern of 7 : it provides $23 \%$ more capacity for scenario II and 3\% more capacity for scenario III. In fact, the number of pilots is reduced (divided by 7) in the reuse 7 because they have to be shared between the all noninterfering cells belonging to this pattern, while, in the case of reuse pattern of 3 , the number of pilots is divided only by 3 . This means that multicell massive MIMO networks with a pilot reuse pattern of 3 can serve more users than those with a reuse pattern of 7.

The results obtained with our proposed method show that a massive MIMO BS (equipped with 256 antennas) is 200 times more energy-efficient while consuming 8 times less power, compared to a LTE BS. These results are comparable to those obtained in the other previous works in $[13,28$, 32] which investigated the energy efficiency and the power consumption of the massive MIMO BS. In [13], a large-scale antenna system (LSAS) equipped with 64 service antennas 
has been investigated in dense urban area. The LSAS can simultaneously serve 15 users, while being almost 1000 times more energy-efficient than a typical LTE BS. The study of [32] shows that a massive MIMO system with 100 and 400 antennas, operating at $3.7 \mathrm{GHz}$ (with $20 \mathrm{MHz}$ bandwidth), consumes 6.5 times and 12 times less power than the traditional LTE network, respectively. Finally, in [28], the authors proposed a power consumption model for the LSAS base stations which can reduce the power consumption of the entire system by a factor of 10 , compared to $4 \mathrm{G}$.

\section{Conclusion}

In this study, we propose an optimal low power design of a massive MIMO network at $3.75 \mathrm{GHz}$ using a capacitybased network deployment tool. In addition to the $4 \mathrm{G}$ reference scenario, two other scenarios have been considered, a crowded one with limited mobility of the users and an urban information society, less crowded but with high mobility. We show that the increase of massive MIMO BS antennas can achieve the same performance of a $4 \mathrm{G}$ reference network in terms of coverage while consuming 8 times less power and providing 200 times more capacity. We also show that massive MIMO is well suited for crowded scenarios where limited mobility of the users is required. The increase of antennas in that case helps provide uniformly good services to 3 times more users simultaneously, compared to the information society scenario. The pilot reuse pattern of 3 appears to be the optimum one to ensure a trade-off between high capacity and low power consumption requirements.

\section{Data Availability}

The data (figures) used to support the findings of this study are included within the article, in the tables. Further details can be provided upon request.

\section{Conflicts of Interest}

The authors declare that there are no conflicts of interest regarding the publication of this paper.

\section{Acknowledgments}

Margot Deruyck and Emmeric Tanghe are postdoctoral fellows of the FWO-V (Research Foundation-Flanders).

\section{References}

[1] T. L. Marzetta, "Noncooperative cellular wireless with unlimited numbers of base station antennas," IEEE Transactions on Wireless Communications, vol. 9, no. 11, pp. 3590-3600, 2010.

[2] F. Boccardi, R. W. Heath, A. Lozano, T. L. Marzetta, and P. Popovski, "Five disruptive technology directions for 5G," IEEE Communications Magazine, vol. 52, no. 2, pp. 74-80, 2014.

[3] E. G. Larsson, O. Edfors, F. Tufvesson, and T. L. Marzetta, "Massive MIMO for next generation wireless systems," IEEE Communications Magazine, vol. 52, no. 2, pp. 186-195, 2014.
[4] E. Björnson, E. G. Larsson, and T. L. Marzetta, "Massive MIMO: Ten myths and one critical question," IEEE Communications Magazine, vol. 54, no. 2, pp. 114-123, 2016.

[5] T. L. Marzetta, E. G. Larsson, H. Yang, and H. Q. Ngo, Fundamentals of Massive MIMO, Cambridge University Press, 2016.

[6] W. Tan, M. Matthaiou, S. Jin, and X. Li, "Spectral Efficiency of DFT-Based Processing Hybrid Architectures in Massive MIMO," IEEE Wireless Communications Letters, 2017.

[7] W. Tan, G. Xu, E. De Carvalho, M. Zhou, L. Fan, and C. Li, "Low Cost and High Efficiency Hybrid Architecture Massive MIMO Systems Based on DFT Processing," Wireless Communications and Mobile Computing, vol. 2018, pp. 1-11, 2018.

[8] W. Tan, S. Jin, C.-K. Wen, and T. Jiang, "Spectral efficiency of multi-user millimeter wave systems under single path with uniform rectangular arrays," EURASIP Journal on Wireless Communications and Networking, vol. 2017, no. 1, 2017.

[9] B. Thors, A. Furuskar, D. Colombi, and C. Tornevik, "TimeAveraged Realistic Maximum Power Levels for the Assessment of Radio Frequency Exposure for 5G Radio Base Stations Using Massive MIMO," IEEE Access, vol. 5, pp. 19711-19719, 2017.

[10] L. Chiaraviglio, A. S. Cacciapuoti, G. di Martino et al., "Planning 5G Networks under EMF Constraints: State of the Art and Vision," IEEE Access, pp. 1-1.

[11] P. Baracca, A. Weber, T. Wild, and C. Grangeat, "A statistical approach for RF exposure compliance boundary assessment in massive MIMO systems," in Proceedings of the 22nd International ITG Workshop on Smart Antennas, WSA, pp. 1-6, Bochum, Germany, 2018.

[12] E. Bjornson, E. G. Larsson, and M. Debbah, "Massive MIMO for Maximal Spectral Efficiency: How Many Users and Pilots Should Be Allocated?" IEEE Transactions on Wireless Communications, vol. 15, no. 2, pp. 1293-1308, 2016.

[13] H. Yang and T. L. Marzetta, "Total energy efficiency of cellular large scale antenna system multiple access mobile networks," in Proceedings of the 2013 IEEE Online Conference on Green Communications, OnlineGreenComm 2013, pp. 27-32, October 2013.

[14] MAMMOET, "Massive MIMO for efficient transmission: Deliverables 1.1, systems scenarios and requirements specifications, 2014".

[15] H. Yang and T. L. Marzetta, "Performance of conjugate and zeroforcing beamforming in large-scale antenna systems," IEEE Journal on Selected Areas in Communications, vol. 31, no. 2, pp. 172-179, 2013.

[16] M. Karlsson and E. G. Larsson, "On the operation of massive MIMO with and without transmitter CSI," in Proceedings of the 2014 15th IEEE International Workshop on Signal Processing Advances in Wireless Communications, SPAWC 2014, pp. 1-5, Canada, June 2014.

[17] N. Akbar, N. Yang, P. Sadeghi, and R. A. Kennedy, "Multi-Cell Multiuser Massive MIMO Networks: User Capacity Analysis and Pilot Design," IEEE Transactions on Communications, vol. 64, no. 12, pp. 5064-5077, 2016.

[18] X. Gao, O. Edfors, F. Tufvesson, and E. G. Larsson, "Massive MIMO in Real Propagation Environments: Do All Antennas Contribute Equally?" IEEE Transactions on Communications, vol. 63, no. 11, pp. 3917-3928, 2015.

[19] S. Q. Jabbar and Y. Li, "Analysis and evaluation of performance gains and tradeoffs for massive MIMO systems," Applied Sciences (Switzerland), vol. 6, no. 10, 2016. 
[20] R. Chataut and R. Akl, "Optimal pilot reuse factor based on user environments in 5G Massive MIMO," in Proceedings of the 2018 IEEE 8th Annual Computing and Communication Workshop and Conference (CCWC), pp. 845-851, Las Vegas, NV, January 2018.

[21] H. Q. Ngo, E. G. Larsson, and T. L. Marzetta, "Massive MUMIMO downlink TDD systems with linear precoding and downlink pilots," in Proceedings of the 51st Annual Allerton Conference on Communication, Control, and Computing, Allerton 2013, pp. 293-298, USA, October 2013.

[22] T. Van Chien, E. Bjornson, and E. G. Larsson, "Joint power allocation and user association optimization for massive MIMO systems," IEEE Transactions on Wireless Communications, vol. 15, no. 9, pp. 6384-6399, 2016.

[23] M. Matalatala, M. Deruyck, E. Tanghe, L. Martens, and W. Joseph, "Performance evaluation of $5 \mathrm{G}$ millimeter-wave cellular access networks using a capacity-based network deployment tool," Mobile Information Systems, vol. 2017, Article ID 3406074 , 11 pages, 2017.

[24] M. Deruyck, W. Joseph, E. Tanghe, and L. Martens, "Reducing the power consumption in LTE-Advanced wireless access networks by a capacity based deployment tool," Radio Science, vol. 49, no. 9, pp. 777-787, 2014.

[25] METIS project, "In scenarios, requirements and KPIs for 5G mobile and wireless system, deliverable D1.1, 2013".

[26] Ericsson, "Ericsson mobility report, 2015".

[27] C. Desset, B. Debaillie, and F. Louagie, "Towards a flexible and future-proof power model for cellular base stations," in Proceedings of the 24th Tyrrhenian International Workshop on Digital Communications - Green ICT (TIWDC), pp. 1-6, Tucson, AZ, USA, September 2013.

[28] C. Desset, B. Debaillie, and F. Louagie, "Modeling the hardware power consumption of large scale antenna systems," in Proceedings of the 2014 IEEE Online Conference on Green Communications (Online Greencomm), pp. 293-298, Monticello, IL, USA, November 2014.

[29] L. M. Correia, D. Zeller, O. Blume et al., "Challenges and enabling technologies for energy aware mobile radio networks," IEEE Communications Magazine, vol. 48, no. 11, pp. 66-72, 2010.

[30] W. Vereecken, W. Van Heddeghem, M. Deruyck et al., "Power consumption in telecommunication networks: overview and reduction strategies," IEEE Communications Magazine, vol. 49, no. 6, pp. 62-69, 2011.

[31] W. Tan, D. Xie, J. Xia, W. Tan, L. Fan, and S. Jin, "Spectral and Energy Efficiency of Massive MIMO for Hybrid Architectures Based on Phase Shifters,' IEEE Access, vol. 6, pp. 11751-11759, 2018.

[32] C. Desset and B. Debaillie, "Massive MIMO for energyefficient communications," in Proceeding of the 46th European Microwave Conference, pp. 138-141, London, UK, October 2016. 


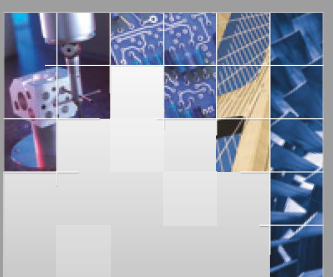

\section{Enfincering}
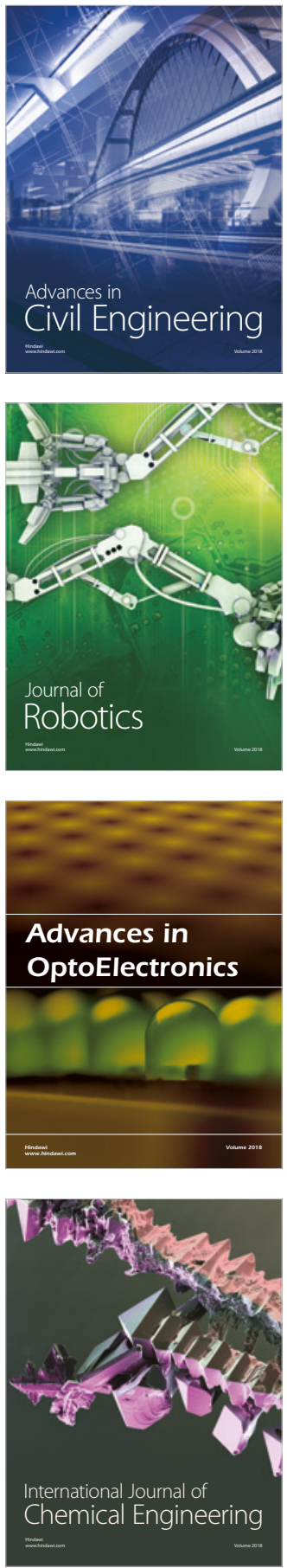

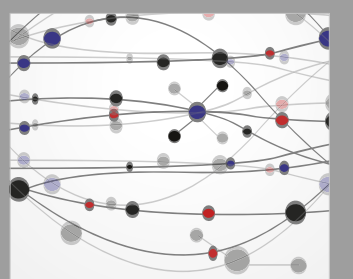

\section{Rotating \\ Machinery}

The Scientific World Journal

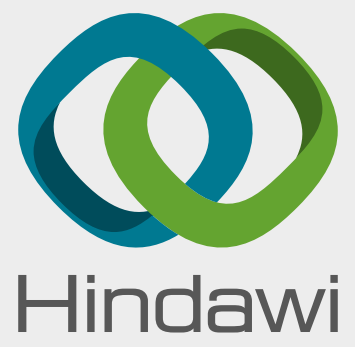

Submit your manuscripts at

www.hindawi.com
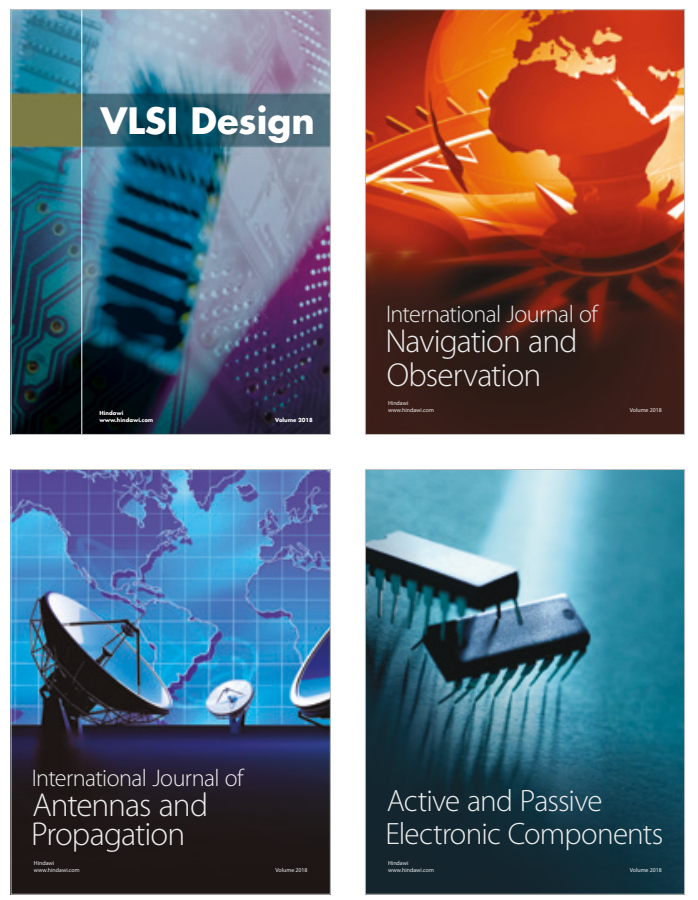
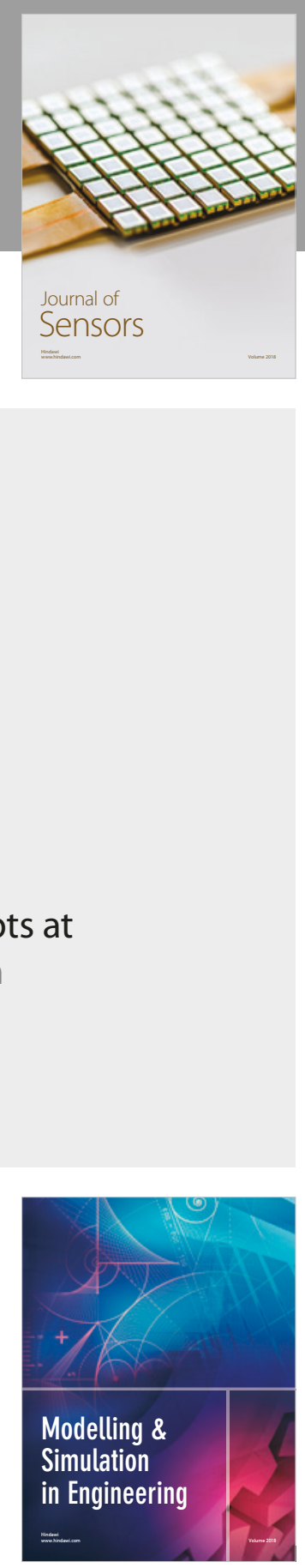

\section{Advances \\ Multimedia}
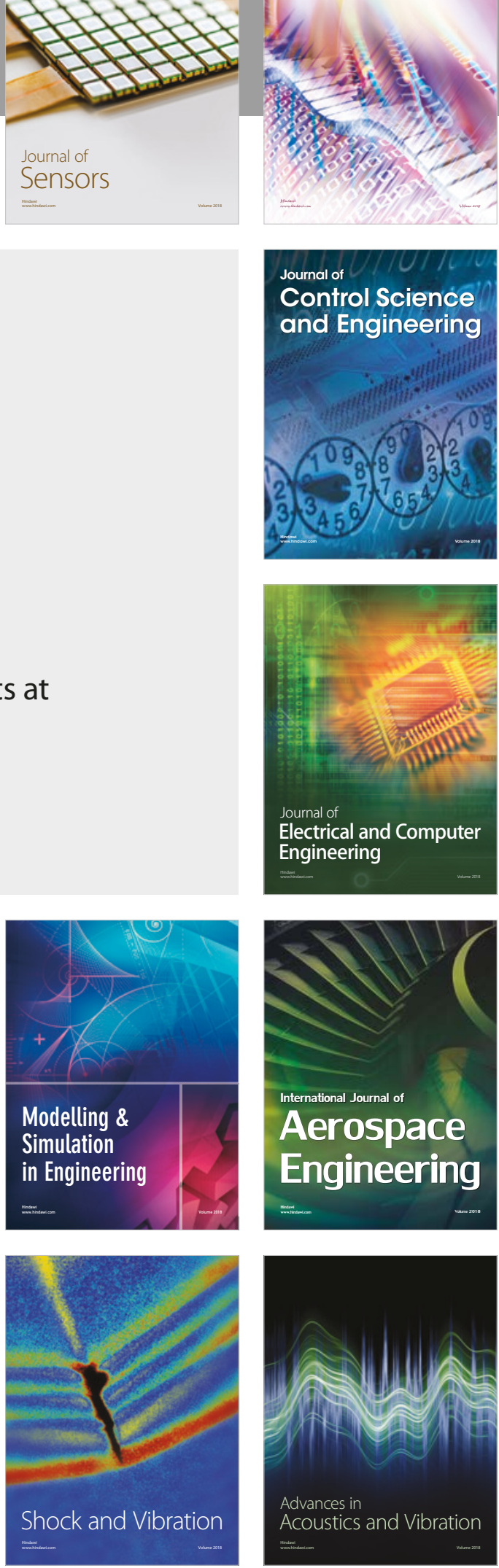